\section{Síndrome de dolor miofascial. Revisión de la literatura a propósito de un caso clínico}

\author{
Myofascial pain syndrome. Literature \\ update on the subject of a clinical case
}

\section{Resumen}

En el presente artículo se resume una evaluación literaria de los últimos 17 ańos en relación al síndrome de dolor miofascial en el cual se recopila la información más relevante para su diagnóstico, además se presenta un caso clínico en el cual se pudo determinar la etiología psicológica que comenzó el trastorno patológico. Se presenta el caso de un paciente con disminución en la apertura bucal, mialgia y puntos gatillo en los músculos masetero y temporal. Se diagnosticó síndrome de dolor miofascial posterior a realizar pruebas musculares, exámenes radiográficos y de laboratorio con la finalidad excluir etiologías sistémicas. El tratamiento indicado consistió en la aplicación de una férula oclusal, fisioterapia, relajantes musculares, corticoesteroides, antiinflamatorios y la interconsulta con un psicólogo. En la primera consulta se midió una apertura bucal de 1,2 cm, en la segunda cita 1,6 cm, en la última consulta se registró una apertura máxima de 4,8 cm después del tratamiento farmacológico, en conjunto con el profesional en psicología. Se concluye que los problemas psicológicos tienen una gran repercusión en el sistema gnatológicoyquer esulta de gran apo yo la inter consulta psicológica.

Palabras clave: Síndrome de la disfunción de articulación temporomandibular; Miositis; Hipertonía muscular; Derivación y consulta; Síndromes del dolor miofascial; Puntos disparadores; Placa motora.

\begin{abstract}
This article summarizes a literature evaluation of the past 17 years in relation to the myofascial pain syndrome in which the most relevant information for a diagnosis is collected, also presents a clinical case in which psychological etiology that began the pathological disorder could be determined. The case of a patient with decrease in the mouth opening, myalgia and points trigger muscle masseter and temporal is presented. Pain myofascial was diagnosed after muscle testing, radiographic and laboratory exams in order to exclude systemic etiologies. The indicated treatment consisted in the application of a splint occlusal, physiotherapy, relaxing muscles, corticosteroids, anti-inflammatories and the consultation with psychologist. In the first appointment an oral opening of 1.2 $\mathrm{cm}$ was recorded, in the second appointment the record was $1.6 \mathrm{~cm}$, in the last query the record was $4.8 \mathrm{~cm}$ after drug treatment in conjunction with professional in psychology. It is concluded that psychological problems has a major impact in gnatologic system and psychological consultation turned out great support for this syndrome.
\end{abstract}

Keywords: Temporomandibular joint dysfunction syndrome; Myositis; Muscle hypertonia; Referral and consultation; Miofascial pain syndromes; Trigger points; Motor endplate.

\section{Caso Clínico}

Cristóbal Landa Román ${ }^{1, a}$, Francisco Javier Gómez Pamatz 2a,b

${ }^{1}$ Universidad Michoacana de San Nicolás de Hidalgo. Michoacán, México.

2 Servicio de Odontopediatría. Hospital Infantil de Morelia.

a Cirujano Dentista.

b Cirujano Maxilofacial.

Correspondencia:

Cristóbal Landa Román

Correo electrónico: clr_31@hotmail.com H. Colegio Militar \#85. Col. Chapultepec sur c.p. 58260. Morelia, Michoacán, México.

\section{Coautor:}

Francisco Javier Gómez Pamatz

fgomezpamatz@yahoo.com.mx

Conflicto de intereses: Los autores declaran no tener conflictos de interés.

Fuente de financiamiento: Autofinanciado

Fecha de recepción: 24/06/17

Fecha de aceptación: 06/08/17 


\section{Introducción}

En 1934 el otorrinolaringólogo James B Costen reportó 11 casos en pacientes con alteraciones atípicas de artritis temporomandíbular, en los cuales determinó que las alteraciones del sistema dentario eran responsables de diversos síntomas de los oídos. En base a su experiencia lo denominó "Trastorno de la articulación temporomandíbular", con el tiempo recibiría el nombre de Síndrome de Costen ${ }^{1,2}$.

No fue hasta el año de 1992 que el doctor David G. Simons, en conjunto con la doctora Janet Travell, describieron un proceso patológico regional no inflamatorio que se manifiesta en cualquier músculo esquelético del cuerpo, y se presenta con un comportamiento agudo o crónico, primario o secundario, prevalente, incapacitante, subdiagnosticado y por consiguiente, poco tratado. Esto sucede debido a que no está acompañado de alteraciones estructurales detectables en las pruebas de gabinete o en estudios de laboratorio, estas alteraciones fueron denominadas "puntos gatillo", caracterizados por ser zonas hipersensibles, palpables en el tejido muscular y que poseen la capacidad de producir dolor en zonas distantes a su localización ${ }^{3-7}$.

Con la finalidad de esclarecer su etiología, los doctores redactaron la hipótesis integrada, la cual está basada en una despolarización anormal de la placa motora por mecanismos presinápticos, sinápticos y postsinápticos, es decir, a una excesiva liberación de acetilcolina causada por un defecto de la enzima acetilcolinesterasa y aumento de la actividad del receptor nicotínico en la membrana muscular postsináptica, generando un potencial de acción y contracción muscular, al mismo tiempo que los sarcómeros, severamente acortados del centro de los nodos y de los discos de contracción, provocan una hipoxia severa local; produciendo a su vez, isquemia que resulta en una actividad continua de contracción muscular máxima ${ }^{4,5,7}$.

En un contexto anatómico, podemos definir al sistema complejo bicondíleo ginglimo artrodial como una entidad única en el cuerpo humano capaz de cumplir las necesidades comunicativas y alimenticias cotidianas, el cual, para lograr desempeñar sus funciones de modo armónico, debe cumplir con los rangos de movilidad que indicó Pérez ${ }^{8}$ en el 2014, cumpliendo una apertura activa de 35 a $55 \mathrm{~mm}$, una apertura mínima funcional de 25 a $35 \mathrm{~mm}$, protrusión de 3 a $6 \mathrm{~mm}$, retrusión de 3 a 4 $\mathrm{mm}$ y una lateralidad de 10 a $15 \mathrm{~mm}$.

Estos rangos de movilidad se pueden ver alterados en respuesta a un proceso inflamatorio que perturba la integridad de las estructuras que conforman la articulación. Y se pueden definir como una entidad nosológica funcional del sistema craneomandibular que se divide en dos grupos; los musculares y los propios de la articulación. Debido a que los dos grupos presentan la misma inervación por el $\mathrm{V}$ par craneal y una sintomatología similar, se vuelve complicado diagnosticarlos de manera independiente ${ }^{9-11}$. Los trastornos temporomandibulares como el síndrome de dolor miofascial se pueden presen- tar en articulaciones sin alteraciones previas y suelen ser el 50-75\% de las causas de atención odontológica a nivel mundial ${ }^{12-15}$, por lo cual se considera un dolor común pero incomprendido que involucra el dolor referido por los puntos gatillo en el musculo esquelético ${ }^{16,17}$.

López ${ }^{18}$ señaló que los pacientes bajo tratamiento de Ortodoncia muestran menor prevalencia a las disfunciones temporomandibulares.

En la recopilación bibliográfica se encontró diversas nomenclaturas basadas en su fisiopatología y anatomopatología, como son los epónimos de: síndrome de dolor y disfunción miofascial; disfunción dolorosa de la articulación temporomandibular, síndrome de disfunción craneomandibular, síndrome de fatiga crónica y síndrome de Costen ${ }^{9,11,19-22}$.

El presente artículo consiste en una evaluación literaria de los trastornos temporomandibulares en los últimos 17 años, enfocado en recopilar datos de mayor importancia que permitan educar al personal clínico y que estos ofrezcan al paciente un tratamiento a sus alteraciones psicosomáticas. Es importante destacar la falta de material literario en Latinoamérica que relacione el síndrome de dolor miofascial con una etiología psicológica, lo cual fue analizado anteriormente en un caso clínico que fue presentado acerca de un paciente que fue tratado en primera instancia farmacológicamente y que este no obtuvo resultados favorables sino hasta acudir a interconsulta con un psicólogo.

\section{Etiología}

Se denomina síndrome de dolor miofascial a las mialgias de etiología desconocida ${ }^{1,12}$ que se pueden relacionar con factores biomecánicos de sobrecarga o sobreutilización muscular generada por microtraumatismos repetitivos ${ }^{3}$. Desde el punto vista de la salud pública, podemos definir los trastornos temporomandibulares como una discapacidad psicológica, social y cultural que afecta la salud general del individuo, generando un nivel de tensión muscular que con la presencia de interferencias oclusales, altera el equilibrio del sistema gnático, desencadenando una disfunción craneomandibular ${ }^{23}$.

López ${ }^{18}$ en el 2004 refirió que existen dos factores predisponentes para las alteraciones temporomandibulares; uno relacionado a las interferencias oclusales y el otro con factores psicológicos.

Sin embargo, se ha demostrado la existencia de factores secundarios como lo son: traumatismos, alteraciones en el crecimiento, cambios hormonales, neoplasias, trastornos autoinmunitarios, infecciones, cansancio o espasmos en los músculos de la masticación e hiperactividad muscular; así como debido a factores locales o sistémicos.

Los factores locales, como el bruxismo o la apertura excesiva de la boca; afectan a las estructuras masticatorias. Mientras que entre los factores sistémicos se encuentra el estrés, la edad, sexo, predisposición genética, la alimentación, así como alteraciones en la articulación tem- 
poromandibular apreciables en los ligamentos, dientes y los músculos de la masticación ${ }^{9-11,24,25}$.

De igual manera, Cabrera y cols. formulan que "los trastornos se deben a estrés y una interferencia oclusal grosera" 26.

Sierra ${ }^{12}$ expresó en el 2014 que existen factores de riesgo que conllevan a los trastornos temporomandibulares y se dividen en seis tipos:

- Infecciones por proximidad: otitis media, otitis externa, mastoiditis, parotiditis y rinosinusopatias.

- Infecciones sistémicas: sífilis, tuberculosis, gonorrea, fiebre tifoidea, neumonía y fiebre reumática.

- Enfermedades sistémicas: artritis reumatoidea, fibromialgia, espondilitis y esclerosis múltiple.

- Alteraciones intraauriculares: del complejo cóndilo-disco, desplazamiento discal anterior, luxación con reducción, luxación sin reducción, sinovitis, capsulitis y retrodiscitis.

- Traumatismos: para su estudio se divide en macro-traumatismos que engloba los golpes directos, y el micro-traumatismos correspondiente al bruxismo.

- Enfermedades psiquiátricas: tensión emocional y neurosis.

Haciendo énfasis en el sexto inciso señalado por Sierra, se analizará la etiología del estrés que se ve implicado en las alteraciones sistémicas que perturban las funciones masticatorias. En respuesta a los trastornos emocionales, el centro emocional del cerebro genera cambios en el núcleo paraventricular del hipotálamo que contiene neuronas involucradas con la respuesta al estrés, las cuales son responsables de la regulación neuroendocrina, autonómica y conductual. Dichas neuronas proyectan a los sitios de control la respuesta autonómica y al sistema límbico, generando una respuesta de ansiedad, liberando neurohormona de acetilcolina o corticotropina al sistema porta que conecta al hipotálamo con la adenohipófisis, estimulando la liberación de la hormona acetilcolina al torrente sanguíneo. El sistema reticular y el sistema límbico son los responsables del estado emocional del individuo; estos, en conjunto con la producción hormonal de la hipófisis, afectan la actividad muscular por medio de las vías gammaeferentes ${ }^{27,28}$.

La premisa etiología ha sido expresada por autores como Okeson ${ }^{28}$, quien señala que "el estrés emocional desempeña un papel importante en los trastornos temporomandibulares".

Poveda y cols. establecieron la similitud entre las manifestaciones de dolor crónico de la articulación temporomandibular, desde el punto de vista psicosocial y conductual ${ }^{29}$.

\section{Clasificación}

Okeson $^{28}$ clasificó los desórdenes musculares en cinco tipos:

- Co-contractura protectora: es la primera respuesta del sistema nervioso central ante una agresión muscular, es una respuesta no patológica.

- Dolor muscular local: denominado mialgia no inflamatoria, es un trastorno que se caracteriza por la liberación de sustancias algógenicas.

- Dolor miofacial: trastorno inflamatorio de la musculatura.

- Mioespamos: contracción tónica muscular inducida por el sistema nervioso central.

- Mialgia por punto gatillo: caracterizado por áreas locales de bandas de tejido muscular duro e hipersensible.

\section{Fisiopatología}

La liberación excesiva de acetilcolina produce cambios en el metabolismo oxidativo, lo que conlleva a la disminución de los sustratos energéticos produciendo la secreción de sustancias algógenas como bradicinina, histamina y sustancia P generando una adaptación protectora ante las cargas y hábitos parafuncionales en el músculo esquelético ${ }^{15,19,22}$.

\section{Signos y síntomas}

Los síntomas característicos de los trastornos temporomandibulares son: bruxismo, dolor sordo y constante, el cual se presenta en el reposo y que incrementa al iniciar movimientos funcionales. Al momento de la palpación se encuentran las bandas de los músculos tensas, hipertónicas e hipersensibles con presencia de puntos gatillo. La limitación biomecánica se presenta de dos formas: una con disminución en la velocidad y otra con desviación de la línea media; siguiendo una inclinación al lado afectado, apnea obstructiva del sueńo, cefalea y chasquido articular. Sin embargo, el signo más notorio es la atrición sobre las superficies incisales de los órganos dentales anteriores 9,10,30-32. Es importante considerar que las mialgias son producto de las miositis; pero esto no significa que siempre se presente dolor en sus etapas iniciales, puesto que muchas veces los pacientes presentan alteraciones hasta etapas tardías ${ }^{11}$.

\section{Diagnóstico}

El diagnostico está enfocado en la anamnesis, la exploración física y una valoración adecuada de los puntos gatillo en los que el paciente refiere dolor activo durante la palpación ${ }^{4,22}$.

La exploración física extra-oral se realiza de dos maneras, la primera midiendo la apertura bucal que debe ser de 40 a $45 \mathrm{~mm}^{1}$ y la segunda se realiza solicitando al paciente que apriete los dientes mientras se pinzan con 
los dedos los músculos de la masticación, buscando la presencia de puntos gatillo en las bandas musculares.

Para determinar el grado de afección en la apertura bucal se mide la distancia entre los bordes incisales de los incisivos superiores e inferiores, cualquier variación en la limitación de la apertura bucal llevará a realizar la prueba de sentido final ("end feel") ${ }^{33}$, en la cual se solicita al paciente que realice la apertura máxima posible hasta que inicie una sensación dolorosa. Se sostiene el borde mentoniano con el dedo pulgar en su parte superior y la falange del dedo índice en el cuerpo de la mandíbula, forzando la apertura, si se logra aumentar el ángulo de apertura entonces se trata de un problema muscular, pero si no se obtiene un dato positivo, entonces se trata de un "end feel" duro, lo cual indica que la etiología es propia de la articulación.

Durante la exploración física se encontrará al paciente con sensibilidad al tacto en el cuero cabelludo, lagrimeo o sequedad en los ojos, palidez o enrojecimiento de los tejidos, enrojecimiento de las conjuntivas y secreción nasal tipo alergia ${ }^{24}$.

Moreno y Escobar ${ }^{34}$ señalan tres criterios de diagnóstico:

- Dolor sordo agravado por la función mandibular

- Puntos gatillo

- Reducción del 50\% del dolor con la infiltración de anestésico intramuscular

Rodríguez y Sánchez ${ }^{10}$ sugieren el interrogatorio de la sociedad americana del dolor orofacial, el cual basa el diagnóstico en una anamnesis y una exploración física. Se debe incluir en la historia clínica para confirmar el diagnóstico de un desorden temporomandibular.

- ¿Tiene dificultad, dolor o ambos al abrir la boca, por ejemplo, al bostezar?

- ¿Se ha quedado alguna vez su mandíbula atascada, bloqueada o desencajada?

- ¿Tiene dificultad, dolor o ambos cuando mastica, habla o mueve la mandíbula?

- ¿Ha notado ruidos en las articulaciones mandibulares?

- ¿Nota frecuentemente rigidez, tirantez o cansancio en la mandíbula?

- ¿Siente dolor alrededor de los oídos, sienes o mejillas?

- ¿Tiene frecuentes dolores de cabeza, cuello o dientes?

- ¿Ha tenido recientemente algún traumatismo en la cabeza, cuello o mandíbula?

- ¿Ha notado algún cambio reciente en su forma de morder?
- ¿Ha sido previamente tratado de algún dolor cervicofacial inexplicable o por un problema de la ATM?

\section{Características}

La triada sintomática se caracteriza por dolor en el área preauricular o muscular, disfunción y/o ruidos auriculares y puntos gatillo que se manifiestan como bandas tensas localizadas en los músculos donde se encuentran nódulos hipersensibles ${ }^{11,30,33}$.

La presencia de tinitus, la perdida de la audición, la otorrea y el prurito pueden ser evidencia de un trastorno temporomandibular ${ }^{35}$.

\section{Diagnóstico diferencial}

Artritis de células gigantes, cefalea tensional y artrosis $9,12,24$.

\section{Estudios y pruebas}

La prueba más simple consiste en la aplicación de dos a tres abate lenguas sobre la superficie oclusal de los molares de manera bilateral y se pide al paciente que cierre la boca consiguiendo con ello disminuir los síntomas.

Como se expresó anteriormente, no existen exámenes de gabinete o laboratoriales que arrojen resultados de interés ante el síndrome de dolor miofascial ${ }^{4}$ únicamente se emplean con la finalidad de realizar un diagnóstico diferencial. Sin contradecir lo anterior, particularmente se puede realizar una polisomnografía para verificar la función patológica muscular ${ }^{9,10}$.

\section{Tratamiento}

Se requiere un método multidimensional enfocado en eliminar los factores desencadenantes asociados a trastornos psicológicos, que permita educar al paciente, en un programa de fisioterapia acompañado de compresas de calor por cuatro días intercalado por frio, férulas orales que permitan un cambio en la dimensión vertical de reposo, lo cual genera por consiguiente una relajación muscular. El apoyo farmacológico se enfoca en el uso de analgésicos, antiinflamatorios, relajantes musculares, corticoesteroides y benzodiacepinas en dosis bajas, los cuales han demostrado ser una opción terapéutica favorable. Con la finalidad de evitar episodios repetitivos, se recomienda la interconsulta con un ortodoncista para corregir la maloclusión. Además, la técnica de biorretroalimentación es un excelente apoyo para que el paciente sea consciente de cómo cambiar la actividad fisiológica muscular 10,12,17,18,22,36,37.

\section{Caso clínico}

Se presenta el caso de un paciente masculino de 26 años de edad que acudió a consulta manifestando odontalgia en el primer molar inferior derecho, asociado a una fractura asociada a un traumatismo mandibular bilateral y a la presencia de una cavidad patológica cariosa. A la anamnesis el paciente no refirió antecedentes heredo-familiares de importancia en relación al padecimiento 
actual. En lo referente a antecedentes personales patológicos nos encontramos con: Operación por fractura en peroné y traumatismo mandibular bilateral de tres semanas de evolución.

Al iniciar la exploración física no se observaron datos patológicos, a la inspección intra-oral se apreció gingivitis generalizada, cálculo dental, caries múltiples y la fractura del primer molar inferior derecho de origen carioso con amplia destrucción de la corona por traumatismo contuso bilateral en el cuerpo de la mandíbula. Al transcurso de los días refirió tener dolor en la rama mandibular, acompańado de la incapacidad para realizar movimientos de apertura bucal. A la exploración física extra-oral se observó una apertura máxima de $2 \mathrm{~cm}$, limitada por una sensación dolorosa en la región maseterina, seguida de un chasquido articular que entonces permitía la apertura bucal de $4 \mathrm{~cm}$. A la auscultación se presentó un chasquido bilateral con los movimientos de apertura bucal y mialgia en el músculo masetero derecho. Con la finalidad de esclarecer un diagnóstico se solicitó una ortopantomografía y estudios sanguíneos para proteína $\mathrm{C}$ reactiva, factor reumatoide, antiestreptolisinas y velocidad de eritrosedimentación. Se realizó una toma de impresión para un guarda oclusal con acetato del número 40 indicando su uso durante $24 \mathrm{~h}$ por dos días. Al tercer día de utilizar la férula, se indicó su uso nocturno cada 12 h. Apoyándonos del uso farmacológico se recetó: metocarbamol de 400 mg, ácido acetilsalicilico de $250 \mathrm{mg}$ cada 8 h por 7 días. Se refirió al ortodoncista para valoración.

Al transcurrir una semana el paciente acudió a consulta para una revisión, en la que indicó haber seguido el tratamiento farmacológico y el uso de la férula oclusal pero que no había seguido las indicaciones de mante-

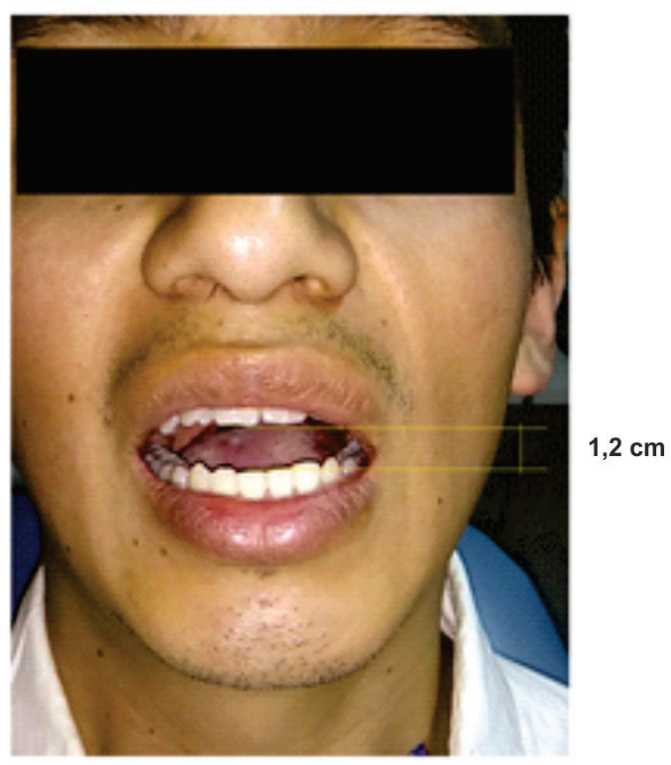

Figura 1. Se solicita al paciente realizar apertura máxima con limitante al iniciar sensación dolorosa. Realizando la medición de los bordes incisales se registró $1,2 \mathrm{~cm}$. ner reposo, generando con ello una disminución de la apertura bucal a $1,2 \mathrm{~cm}$, como se aprecia en la figura 1 . Platicando con el paciente hizo referencia a estarse sintiendo agobiado ante situaciones emocionales que no le permitían conciliar el sueño, también refirió no haberse realizado los estudios solicitados debido a la demanda laboral que tenía, por lo cual se realizó un ajuste farmacológico con: paracetamol de $300 \mathrm{mg}$, clorzoxazona de $250 \mathrm{mg}$ cada $8 \mathrm{~h}$ por 5 días.

Trascurridas dos semanas de inicio del padecimiento se presentó el paciente con la ortopantomografía en la cual se podía observar disminución en el espacio discal de $0,5 \mathrm{~mm}$ de manera bilateral. Como se observa en la figura 2. A la palpación el músculo masetero presentó puntos gatillo mientras que el músculo temporal hipertónico no los presentó. Se registró una apertura máxima de $1,6 \mathrm{~cm}$ (Figura 3). En conjunto a la apertura máxima se procedió a cumplir con la prueba de "end feel" con datos positivos. En los estudios laboratoriales se registraron los resultados de: Proteína C reactiva $<6 \mathrm{mg} / \mathrm{ml}$, factor reumatoide $<8 \mathrm{UI} / \mathrm{ml}$, antiestreptolisinas $<200 \mathrm{UI} / \mathrm{ml}$, velocidad de eritrosedimentación $3 \mathrm{~mm} / \mathrm{h}$. En relación a la información recopilada en los estudios de gabinete y de laboratorio, se pudo esclarecer el diagnóstico positivo de síndrome de dolor miofascial de origen psicosomático, esto mismo basado en que no se presentaron alteraciones propias de las estructuras anatómicas o de discrepancias en los resultados sanguíneos de referencia. El plan de tratamiento se enfocó en instruir al paciente

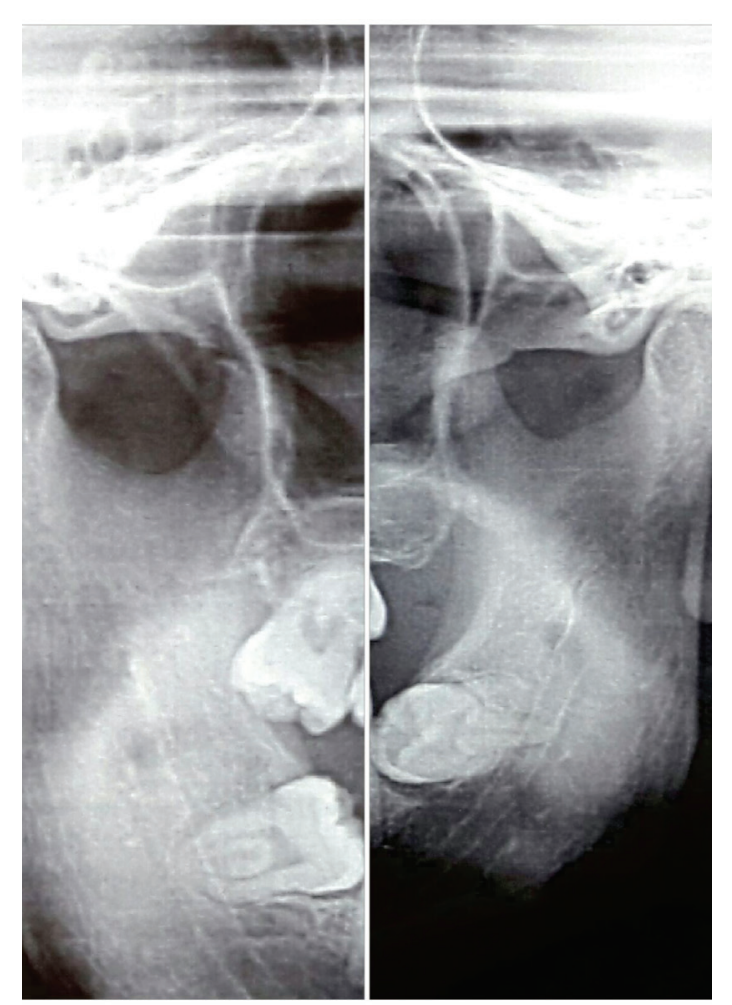

Figura 2. Se observó un comparativo articular radiográfico de una ortopantomografía. Se aprecia disminución del espacio discal articular, desgaste de la eminencia articular del temporal izquierdo, no existe manifestación bilateral de artrosis. 
con fisioterapia enfocada en estiramiento muscular apoyada con abate lenguas sobre el plano oclusal de los molares, continuando con el uso de $24 \mathrm{~h}$ de la férula oclusal por siete días. Sumado a ello se solicitó la aplicación de compresas de calor cuatro veces al día intercalando a frío. Como último, apoyado en la información compartida por el paciente, se indicó la interconsulta con un especialista en psicólogía. Realizando una permuta farmacológica con: Omeprazol $20 \mathrm{mg}$ cada $12 \mathrm{~h}$ por tres semanas, indometacina, betametasona, metocarbamol $25 / 0,75 / 215 \mathrm{mg}$ cada $12 \mathrm{~h}$ por tres semanas.

A la tercera semana el paciente acudió a consulta refiriendo que había asistido con el especialista en psicología obteniendo resultados favorables. A la exploración extra-oral se midió una apertura bucal de 4,8 cm (Figura 4).

\section{Discusión}

La comunicación entre odontólogo y psicólogo fue importante para determinar que la etiología del trastorno era psicosomático, la especialista en psicología informó que realizó una prueba denominada: "Escala del estrés percibido de Cohen, Kamarak y Mermelstein en su versión Mexicana de González y Landero" determinando un diagnóstico de: Trastorno de ansiedad generalizado, por el cual se encontraba ya en tratamiento terapéutico ${ }^{38}$.

Basado en la hipótesis integrada de Simons y cols. ${ }^{5}$ en que las hormonas provenientes de la hipófisis actúan directamente sobre los puntos gatillo mismo que forman parte del síndrome del dolor miofascial, podemos determinar que el trastorno patológico por el que cursaba el paciente se originó por alteraciones psicológicas que se resolvieron al recurrir con el especialista. El tratamiento farmacológico aportó los requerimientos necesarios para

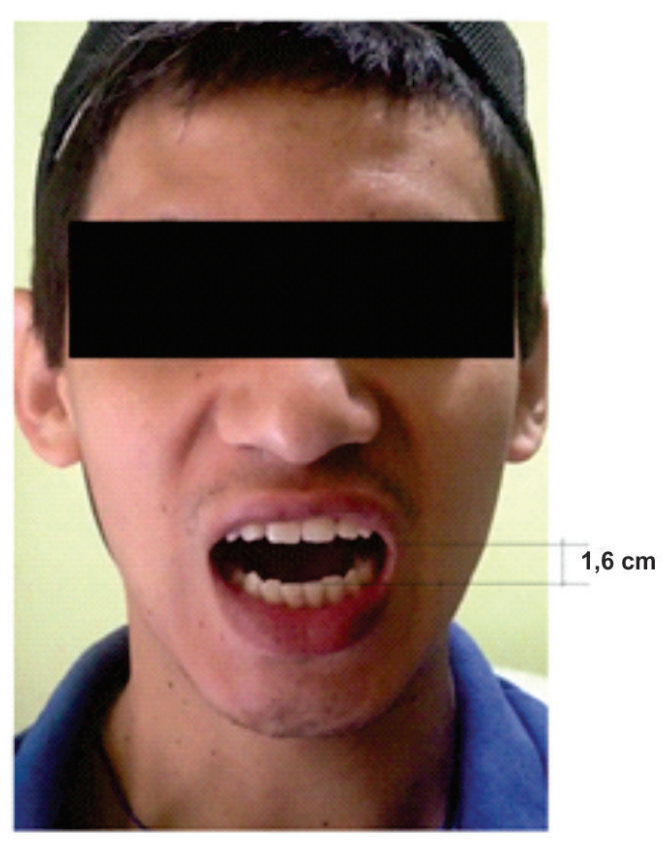

Figura 3. Se aprecia un incremento de la apertura máxima. A la medición se obtiene $1,6 \mathrm{~cm}$, desviación de línea media al lado izquierdo producto de contracción muscular. resolver el trastorno pero era necesario atender el factor etiológico principal que disminuía el aporte de oxígeno al músculo.

La Touche menciona que diversos trastornos que afectan el sistema craneomandibular pueden tener características muy similares, por lo cual determinar un diagnostico a primera instancia resulta complejo ${ }^{30}$.

Peńón y cols. ${ }^{14}$, reportaron que el $67,1 \%$ de los pacientes fueron del sexo femenino, en el $54,8 \%$ de los casos fue del lado derecho y el $13,7 \%$ fue por compromiso articular bilateral. Demostraron también que se presentaba del lado derecho y por actividad funcional asimétrica.

En consulta privada se mantiene un registro de 3:1 en relación al género, con predilección por el género femenino y una función anormal articular del lado derecho. Otras posibles etiologías que comparten una contracción muscular involuntaria son las enfermedades autoinmunes, infecciones virales y bacterianas.

Existen alternativas terapéuticas como modificar los hábitos de vida y evitar alimentos duros o gomas de mascar. Algunos casos fueron tratados con acupuntura, magnetoterapia, ozonoterapia, la apiterapia y la infiltración de toxina botulínica tipo A, infiltrando 250 unidades en cada músculo ${ }^{1,10,13,39,40}$.

Realizar interconsulta es vital en la práctica odontológi$\mathrm{ca}$, no solo entre especialistas de la materia sino también con médicos y psicólogos. Una nueva tendencia que está tomando peso en la práctica odontológica es solicitar radiografía ortopantomográfica al inicio de la consulta, permitiendo así diagnosticar oportunamente alteraciones que se denominan hallazgos radiográficos.

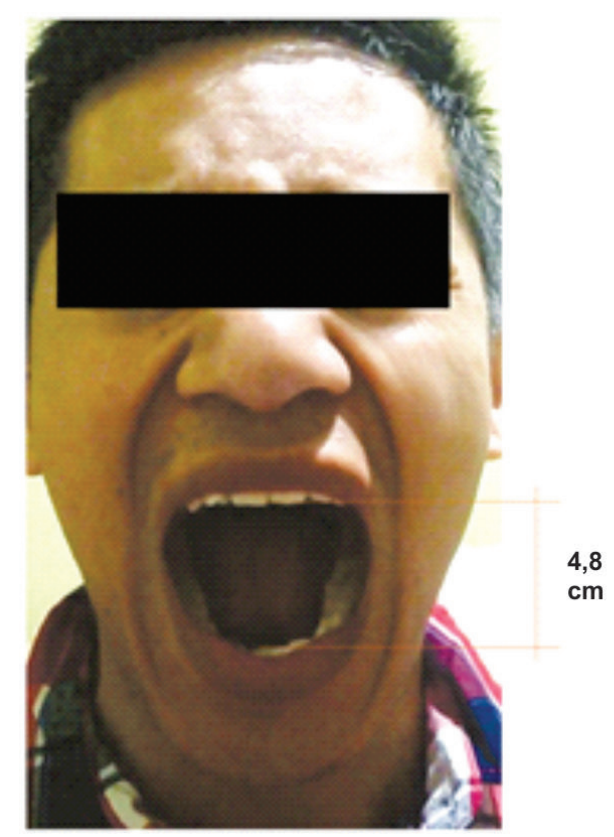

Figura 4. Es notable un incremento de la apertura bucal a 4,8 cm; desviación de $1 \mathrm{~mm}$ de la línea media al lado izquierdo. A la palpación muscular no manifiesta presencia de puntos gatillo. 


\section{Conclusiones}

Los auxiliares de diagnóstico permiten brindar un tratamiento oportuno e interceptivo, minimizando los dańos ocasionados por el bruxismo en los órganos dentales y la hipertrofia muscular que conlleva a limitar los movimientos ideales de la mandíbula.

De los casos tratados en la consulta privada se ha demostrado un pronóstico favorable en los pacientes que acuden a interconsulta psicológica en comparación con los pacientes que no acuden con el especialista en psicología. Demostrando que las patologías de etiología psicosomáticas deben ser tratadas en conjunto y no de manera independiente.

Además de la interconsulta psicológica es necesario recurrir a médicos con la finalidad de tener apoyo en el diagnóstico de enfermedades sistémicas que presentan manifestaciones bucales similares a las psicológicas. Por lo cual, es necesario que el médico solicite los estudios laboratoriales pertinentes para descartar o identificar patologías de etiología autoinmunes, bacteriales y virales tienen como manifestación bucal la contracción muscular y el bruxismo.

La evidencia clínica demuestra que los trastornos temporomandibulares son frecuentes en los pacientes que se encuentran bajo tensión emocional asociada a problemas laborales, familiares y económicos.

\section{Agradecimientos}

A la Arq. Mariana Ferreyra Murillo por su aportación en la traducción literaria.

\section{Referencias bibliográficas}

1. Grau LI, Fernández LK, González G, Osorio NM. Algunas consideraciones sobre los trastornos temporomandibulares. Rev Cubana Estomatol. 2005;42:(3)2-4.

2. Soto CL, Aguirre EI, De la Torre RE. Trastornos temporomandibulares en pacientes con maloclusiones. Rev Cubana Estomatol. 2013;50(4):374-387.

3. Climent J. Bases. Neurofisiológicas del síndrome miofascial. Rehabilitación intervencionista en I'aparell locomotor. 2a ed. Valencia: Lorato. 2011:7-23.

4. Simons D. Revisión de los enigmáticos puntos gatillo miofasciales como causa habitual de dolor y disfunción músculo esqueléticos enigmáticos. Fisioterapia. 2005;27(2):103-120.

5. Simons D, Travell J, Simons L. Dolor y disfunción miofascial. 2a ed. Madrid: Médica Panamericana. 2007.

6. Murillo J, Rodríguez D. Síndrome Miofascial. Rev Med leg Costa Rica. 2016;33(1):3-7.

7. Chavarría SJ. Síndrome de dolor miofascial, diagnóstico y tratamiento. Rev Med de Cos Cen. 2014;71(612):683689.

8. Pérez A. Anatomía y Biomecánica de la región craneofacial y craneomandibular. Madrid: Facultad de ciencias de la salud. 2014.
9. Mehta RN. Síndrome del dolor miofascial - Trastornos odontológicos. Kenilworth: Manual MSD versión para profesionales. 2017.

10. Rodríguez R, Sánchez O. Patología de la articulación temporomandíbular. Nigran: Pontevedra. 2010.

11. Vieira D. Síndrome de la DCM [Internet]. Propdental. 2015 [cited 1 June 2017]. Available from: http://www. propdental.es/blog/odontologia/sindrome-de-la-dcm/

12. Sierra M. Relación de trastornos de la articulación temporomandíbular y sintomatología ótica en paciente adulto del hospital santa Matilde de Madrid cundinamarca en el periodo de enero de 2014 a junio de 2015 [trabajo de investigación]. Universidad de ciencias aplicadas y ambientales facultad de ciencias de la salud. 2016.

13. Gómez-Pombo A, Martínez-Salgado J, Morillas P, García-Rojo B, Cánovas L, Castro M. Tratamiento del Síndrome de Dolor Miofascial con Toxina Botulínica tipo A. Rev Soc Esp Dolor. 20016;13(2):96-102.

14. Peñón VP, Grau LI, Sarracent PH. Caracterización clínica del síndrome de disfunción temporomandibular en el Hospital Universitario "Miguel Enríquez". Rev Cubana Estomatol. 2011;48(4):371-381.

15. Tenas LJ, Forcén BA, Ruiz NM, Royo-Villanova PM. Estudios sobre las asociaciones terapéuticas en el Síndrome de Costen. Rev Eur Odontoestomatol. 2002;14(6):317322.

16. Fricton JR, Kroening R, Haley D, Siegert R. Myofascial pain syndrome of the head and neck: a review of clinical characteristics of 164 patients. Oral Surg Oral Med Oral Pathol Oral Radiol. 1985;60(6):615-623.

17. Dao TT, Lavigne GJ, Charbonneau A, Feine JS, Lund JP. The efficacy of oral splints in the treatment of myofascial pain of the jaw muscles: a controlled clinical trial. Pain. 1994;56(1):85-94.

18. López E. Terceros molares y disfunción temporomandibular en alumnos de la facultad de odontología de la UMSNH [Tesis de licenciatura]. Universidad Michoacana de San Nicolás de Hidalgo. 2004.

19. Ruiz M, Nadador V, Fernández-Aleantud J, Hernández SJ, Riquelme I, Benito G. Dolor de origen muscular: dolor miofascial y fibromialgia. Rev Soc Esp Dolor. 2007;14(1):37-43

20. García de Hombre AM. Trastorno doloroso y vértigo referido al oído: Derivación frecuente al ORL. An Med Interna. 2005;22(2):88-90.

21. Mix SH. Síndrome doloroso de la articulación temporomandibular. Rev Otorrinolaringol Cir Cabeza Cuello. 1996;56(3):143-148.

22. Francisco FH. Síndromes miofasciales. Reumatol Clin. 2009;5(Supl E2):36-39.

23. Garza C. Disfunción temporomandibular en los diferentes tipos de maloclusión [Tesis de maestría]. Universidad Autónoma de Nuevo León Facultad de Salud Pública. 1993.

24. Oclusiondental - P01. Clasificación de TTM [Internet]. Oclusiondental.wikispaces.com. 2017 [cited 17 May 2017]. Available from: https://oclusiondental.wikispaces.com/P01.+Clasificaci\%C3\%B3n+de+TTM. 
25. Jewkes J. Bruxismo y disyunciones temporomandibulares enfoque kinésico [Tesis de licenciatura]. Universidad Fasta. 2011.

26. Cabrera VY, Álvarez LM, Gómez MM, Malcom CM. Oclusión y estrés en el síndrome dolor-disfunción temporomandibular: presentación de un paciente. Rev Arch Med Camagüey. 2009;13(3):311-320.

27. Leira M. Manual de bases biológicas del comportamiento humano. 1era ed. Montevideo: Universidad de la República. 2012.

28. Okenson J. Tratamiento de oclusión y afecciones temporomandibulares. 4ta ed. Madrid: Mosby Harcourt. 2003.

29. Poveda RR, Bagán J, Díaz FJ, Hernández BS, Jiménez SY. Review of temporomandibular joint pathology: Part I: Classification, epidemiology and risk factors. Med Oral Patol Oral Cir Bucal. 2007;12(4):93-97.

30. La Touche R. Diagnóstico clínico de artrosis en la articulación temporomandibular asociado a un síndrome de dolor miofascial. Rev Soc Esp Dolor. 2007;14(7):490493.

31. Taboada AO, Gómez GYL, Taboada AS, Mendoza NVM. Prevalencia de signos y síntomas de los trastornos temporomandibulares en un grupo de adultos mayores. Rev Assoc Dent Mex. 2004;61(4):125-129.

32. Vázquez DE, Cascos RJ, Gay EC. Myofascial pain syndrome associated with trigger points: A literature review. (I): Epidemiology, clinical treatment and etiopathogeny. Med Oral Patol Oral Cir Bucal. 2009;14(10): e494498.
33. Rudd PA, McNeill C. Diagnóstico diferencial de dolor orofacial con especial énfasis en los desórdenes temporomandibulares. Dol Clin Ter 2005;3(10)15-19.

34. Villaseñor-Moreno JC, Escobar- Reyes VH, De la Lanza-Andrade LP, Guizar-Ramírez BI. Síndrome de dolor miofascial. Epidemiologia, fisiopatología, diagnóstico y tratamiento. Rev Esp Med Quir. 2013;18:148-157.

35. Oviedo-Montes AF, Ramblas-Ángeles MP, Ocampo AA. El trastorno craneomandibular no diagnosticado. Cir Cirujanos. 2001;69(5)242-246.

36. Ramírez M. Evaluación de los trastornos temporomandibulares en centros geriátricos de Culiacán, Sinaloa [Tesis de Doctorado]. Universidad de Granada. 2009.

37. Manns A, Miralles R, Santander H, Valdivia J. Influence of the vertical dimension in the treatment of myofascial pain-dysfunction. J Prosthet Dent. 1983;50(5):700709 .

38. González-Ramírez MT, Landero-Hernández R. Confirmación de un modelo explicativo del estrés y de los síntomas psicosomáticos mediante ecuaciones estructurales. Rev Panam Salud Pública. 2008;23(1):7-18.

39. Rodríguez M, Mursulí M, Díaz R, Rodríguez T. Magnetoterapia en el dolor miofacial. Presentación de casos. [Internet]. Bvs.sld.cu. 2011 [cited 2 June 2017]. Available from: http://www.bvs.sld.cu/revistas/gme/pub/ vol.13.(3)_09/p9.html

40. Santana FK, Rey FY, Ricardo RE, Silva CM, Rodríguez HA. Aplicación de la medicina tradicional y natural en las urgencias de prótesis estomatológicas. Rev Arch Méd Camagüey. 2015;19(3):314-324. 